\title{
True incidence of uterine adenomatoid tumors
}

\author{
HIROFUMI NAKAYAMA ${ }^{1}$, HIDEKI TERAMOTO ${ }^{2,3}$ and MITSUE TERAMOTO ${ }^{2,3}$ \\ Departments of ${ }^{1}$ Pathology and Laboratory Medicine and ${ }^{2}$ Obstetrics and Gynecology, Hiroshima General Hospital \\ of West Japan Railway Company, Higashi-ku, Hiroshima 732-0057, Japan
}

Received January 29, 2013; Accepted February 25, 2013

DOI: $10.3892 /$ br.2013.72

\begin{abstract}
Uterine adenomatoid tumors (UATs) are benign tumors of the uterine serosa and myometrium that originate from the mesothelium and forming gland-like structures. This study was conducted in order to determine the true incidence of UATs, which are usually an incidental finding during uterine surgery performed for other causes. UATs may resemble pre-existing vessels and lymphatic ducts, as well as metastatic adenocarcinomas. A total of 199 consecutive surgical operations (134 hysterectomies and 65 uterus-preserving tumor excisions) were performed by a single team of gynecologists and examined by a single attending pathologist, who performed a thorough macro- and microscopic examination of all the specimens. UATs were identified in nine $(5 \%)$ out of the 199 cases [six (5\%) out of the 134 hysterectomies and three $(5 \%)$ out of the 65 uterus-preserving tumor excisions]. Therefore, the true incidence of UATs may be significantly higher than $1 \%$, which is the incidence reported in the presently available literature.
\end{abstract}

\section{Introduction}

Uterine adenomatoid tumors (UATs) are benign tumors of the uterine serosa and myometrium, originating from the mesothelium and forming gland-like structures (1). UATs are incidentally discovered during uterine surgery performed for other causes (1) and may resemble pre-existing capillaries and lymphatic ducts, as well as metastatic adenocarcinomas (2). Therefore, it is important for surgical pathologists to determine the true incidence of UATs. In one of the major textbooks of gynecological pathology (2) it is reported that UATs are

Correspondence to: Dr Hirofumi Nakayama, Department of Pathology and Laboratory Medicine, Hiroshima General Hospital of West Japan Railway Company, 3-1-36 Futabanosato, Higashi-ku, Hiroshima 732-0057, Japan

E-mail: hinakayama-path@umin.ac.jp

Present address: ${ }^{3}$ Department of Obstetrics and Gynecology, Hiroshima City Asa Hospital, Hiroshima, Japan

Key words: uterine adenomatoid tumor, incidence discovered in $\sim 1 \%$ of hysterectomy specimens; however, there is no citation of original studies.

In order to determine the true incidence of UATs, large numbers of consecutive hysterectomy and uterus-preserving tumor excision specimens should be thoroughly examined by a single attending pathologist.

\section{Materials and methods}

Cases. A total of 199 consecutive surgical cases were investigated over a period of 18 months. The cases were as follows: 66 vaginal hysterectomies [43 due to uterine prolapse, 18 due to leiomyomas, three due to adenomyosis and two due to residual high-grade squamous intraepithelial lesions (HSILs)], 26 laparoscopically-assisted vaginal hysterectomies (22 due to leiomyomas and four due to adenomyosis), 31 transabdominal simple hysterectomies (17 due to leiomyomas, 7 due to adenomyosis, one due to atypical endometrial hyperplasia, one due to a large endometrial polyp, two due to severe infection, two due to massive bleeding following delivery and placenta increta and one due to torsion of a benign ovarian cystic tumor), nine radical hysterectomies due to malignant tumors and two total laparoscopically-assisted hysterectomies due to adenomyosis. As summarized in Table I, the major indications for hysterectomy were leiomyomas (57 cases), uterine prolapse (43 cases), adenomyosis (16 cases) and malignant tumors, including residual HSIL and atypical endometrial hyperplasia (18 cases). The remaining 65 patients underwent uterus-preserving tumor excisions (64 laparoscopically-assisted and one transvaginal myomectomies), following clinical diagnosis of large leiomyomas.

The study was approved by the Ethics Committee of the Hiroshima General Hospital of West Japan Railway Company, Higashi-ku, Hiroshima, Japan. Informed consent was obtained from the patients or the patients' families.

Analysis. A single attending pathologist (H.N.) performed a macroscopic examination of the surgical specimens obtained from 199 consecutive cases handled by a single gynecological surgical team, including two attending gynecologists (H.T. and M.T.). The attending pathologist collected tissue samples macroscopically resembling UATs for further histological examination. A diagnosis of UAT was confirmed by immunohistochemical analysis, using monoclonal antibodies to calretinin (clone DAK Calret 1; 
Table I. Relationship between clinical diagnosis and presence of UATs in 199 consecutive gynecological surgical cases.

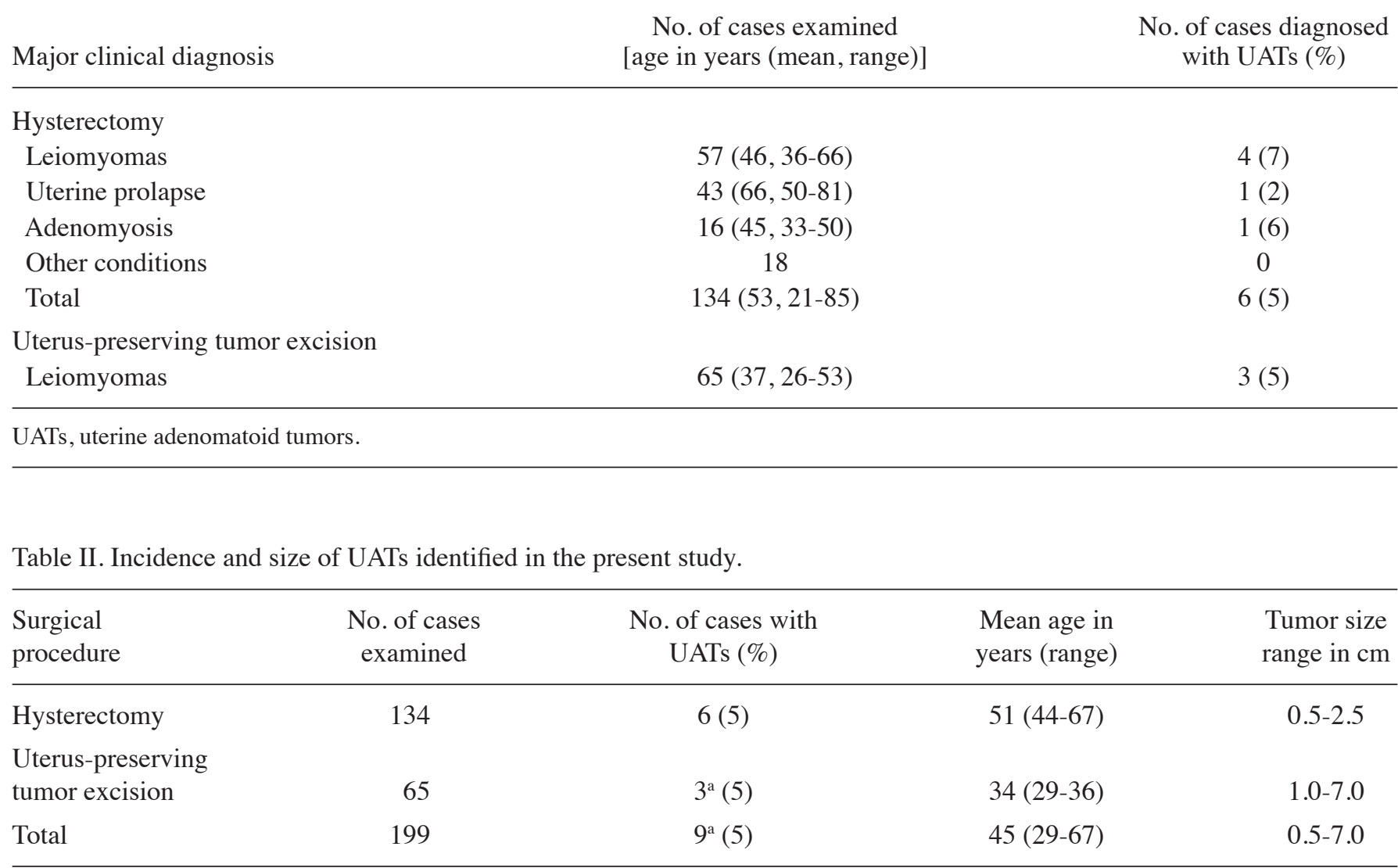

${ }^{a}$ One case (case 4 in Table III) presented with two UATs; therefore, a total of 10 UATs were identified in the present study. UATs, uterine adenomatoid tumors.

Table III. Clinicopathogical characteristics of the nine cases ultimately diagnosed with UATs.

\begin{tabular}{|c|c|c|c|c|c|}
\hline Case no. & $\begin{array}{l}\text { Age } \\
\text { (years) }\end{array}$ & $\begin{array}{l}\text { Preoperative } \\
\text { UAT detection }\end{array}$ & $\begin{array}{l}\text { Other lesions } \\
\text { identified }\end{array}$ & $\begin{array}{l}\text { Surgical } \\
\text { procedure }\end{array}$ & $\begin{array}{l}\text { Size } \\
(\mathrm{cm})\end{array}$ \\
\hline 1 & 45 & Not detected & Leiomyomas & $\mathrm{H}$ & 0.6 \\
\hline 2 & 44 & Misdiagnosed as a leiomyoma & Leiomyomas & $\mathrm{H}$ & 2.5 \\
\hline 3 & 48 & Not detected & Leiomyomas & $\mathrm{H}$ & 0.8 \\
\hline 4 & 36 & $\begin{array}{l}\text { Misdiagnosed as two minor foci } \\
\text { of adenomyosis (two UATs) }\end{array}$ & Leiomyoma & $\mathrm{T}$ & 1.0 \\
\hline 5 & 53 & Not detected & Leiomyomas & $\mathrm{H}$ & 0.5 \\
\hline 6 & 36 & Misdiagnosed as adenomyosis & Leiomyomas & $\mathrm{T}$ & 3.0 \\
\hline 7 & 67 & Not detected & Uterine prolapse & $\mathrm{H}$ & 0.6 \\
\hline 8 & 50 & Not detected & Adenomyosis & $\mathrm{H}$ & 0.8 \\
\hline 9 & 29 & $\begin{array}{l}\text { Misdiagnosed as a leiomyoma } \\
\text { with degenerative change }\end{array}$ & None & $\mathrm{T}$ & 7.0 \\
\hline
\end{tabular}

UATs, uterine adenomatoid tumors; H, hysterectomy; T, uterus-preserving tumor excision.

DakoCytomation, Kyoto, Japan), podoplanin (D2-40; Nichirei Bioscience, Tokyo, Japan), CK5/6 (D5/16B4; DakoCytomation), CK8 (CAM5.2; Becton-Dikinson, Tokyo, Japan), CD34 (QBEnd10; DakoCytomation) and CD31 (JC/70A; DakoCytomation) (3).

\section{Results}

Incidence. As shown in Tables I and II, UATs were identified in nine $(5 \%)$ out of the 199 cases investigated [six $(5 \%)$ out of the 134 cases of hysterectomy and three $(5 \%)$ out of the 65 
cases with uterus-preserving tumor excision]. One out of these nine cases presented with two UATs. Accordingly, a total of 10 UATs were identified during the present study.

Age. As summarized in Table II, the age range of the nine patients diagnosed with UATs was 29-67 years (six hysterectomy patients, 44-67 years; three uterus-preserving tumor excision patients, 29-36 years).

Tumor size. As shown in Table II, the 10 UATs identified in the present study ranged in size from 0.5 to $7 \mathrm{~cm}$; five $(84 \%)$ out of the six UATs that were incidentally discovered in hysterectomy specimens at gross examination were $<1 \mathrm{~cm}$ in diameter, whereas the four $(100 \%)$ UATs identified in the three cases of uterus-preserving tumor excision were $>1 \mathrm{~cm}$ in diameter.

Clinicopathological characteristics of the nine cases diagnosed with UATs. The clinicopathological characteristics are listed in Table III. Among the hysterectomy cases, case 2 presented with numerous nodular lesions $<7.5 \mathrm{~cm}$ in diameter, preoperatively detected by magnetic resonance imaging (MRI) scans; one of these lesions, measuring $2.5 \mathrm{~cm}$ in diameter, was subsequently diagnosed as a UAT; the other lesions were all confirmed as leiomyomas. The remaining five UATs in cases $1,3,5,7$ and 8 were incidentally detected by the attending surgical pathologist.

Among the cases of uterus-preserving tumor excision, the gynecologists detected three nodular lesions by MRI in case 4; one of these three lesions, measuring $6 \mathrm{~cm}$ in diameter, was preoperatively diagnosed as a leiomyoma and the remaining two, measuring $1 \mathrm{~cm}$ in diameter, were suspicious of adenomyosis. The histopathological examination confirmed the diagnosis of a leiomyoma and two UATs, respectively. The clinical diagnosis in case 6 included leiomyomas and a large focus of adenomyosis; all preoperatively diagnosed leiomyomas were histopathologically confirmed as such and the large focus clinically misdiagnosed as adenomyosis was in fact a UAT. Case 9 presented with the largest UAT identified in the present study, which was preoperatively misdiagnosed as a leiomyoma with degenerative change by MRI scans.

\section{Discussion}

As mentioned above, according to one of the major textbooks of gynecological pathology, UATs are identified in $\sim 1 \%$ of hysterectomy specimens (2); this textbook was also cited as a reference in another widely recognized gynecological pathology textbook (4). However, those textbooks included no citation of original studies. To the best of our knowledge, our study is the first to report findings regarding the incidence of UATs, since there are no available data on the subject in the literature.

Total hysterectomy specimens enable pathologists to examine the whole uterus. Therefore, hard data on the true incidence of UATs may only be obtained through the study of total hysterectomy specimens, since all UATs $<1 \mathrm{~cm}$ in diameter were incidentally discovered in hysterectomy specimens. In the present study, the incidence of UATs observed among consecutive hysterectomy cases was identical to that in uterus-preserving tumor excision cases. Among the several uterus-preserving tumor excision procedures, laparoscopically-assisted myomectomy (LAM) is the most widely accepted by gynecologists, as it allows the excision of hundreds of nodular lesions from the myometrium of a single patient. Therefore, we hypothesize that if larger numbers of LAM cases are accumulated and the nodular lesions excised are histopathologically examined, large numbers of small-sized UATs may be incidentally discovered.

The true incidence of UATs may be significantly higher than mentioned in the presently available literature $(2,4)$. Further investigations, including accumulation of hysterectomy as well as LAM cases, are required to confirm the present results.

\section{References}

1. Hendrickson MR, Tavassoli FA, Kempson RL, McCluggage WG, Haller U and Kubik-Huch RA: Mesenchymal tumors and related lesions. In: WHO Classification Tumors of the Breast and Female Genital Organs. Tavasolli FA and Devilee P (eds). IARC, Lyon, pp233-244, 2003.

2. Zaloudek C and Norris HJ: Mesenchymal tumors of the uterus. In: Blaustein's Pathology of the Female Genital Tract. 4th edition. Kurman R (ed). Springer-Verlag, pp487-528, 1994.

3. Sangoi AR, McKenney JK, Scwartz EJ, Rouse RV and Longacre TA: Adenomatoid tumors of the female and male genital tracts: a clinopathological and immunohistochemical study of 44 cases. Mod Pathol 22: 1228-1235, 2009.

4. Rollason TP and Wilkinson N: Non-neoplastic conditions of the myometrium and pure mesenchymal tumors of the uterus. In: Haines and Taylor's Obstetrical and Gynecological Pathology. Fox $\mathrm{H}$ and Wells $\mathrm{M}$ (eds). 5th edition. Churchill Livingstone, Edinburgh, pp497-548, 2003. 